\title{
Modelo Experimental para el Estudio de la Reconstrucción Mandibular. Opciones en Conejos Oryctolagus cuniculus
}

\author{
Experimental Model for the Study of Mandibular Reconstruction. \\ Options in Rabbits Oryctolagus cuniculus
}

\author{
Juan Pablo Alister ${ }^{1,2,3}$; Carlos Veuthey ${ }^{4}$; Francisca Uribe ${ }^{1,2,3}$; Bélgica Vásquez ${ }^{5}$; Mariano del Sol ${ }^{4}$ \& Sergio Olate Sa, $^{1,2,4}$
}

\begin{abstract}
ALISTER, J. P.; VEUTHEY, C.; URIBE, F. ; VÁSQUEZ, B.; DEL SOL, M. \& SERGIO OLATE, S. Modelo experimental para el estudio de la reconstrucción mandibular. Opciones en conejos Oryctolagus cuniculus. Int. J. Morphol., 35(3):1185-1190, 2017.

RESUMEN: El propósito de este estudio fue de evaluar la técnica quirúrgica propuesta para estudios de resección y reconstrucción mandibular en modelos experimentales en conejos. Se utilizaron 7 conejos neozelandeses (Orictolagus cuniculus), en los cuales se realizó la resección del cuerpo mandibular izquierdo, una vez asegurada la movilidad de los segmentos, se reconstruyó con placa de osteosíntesis de titanio de $1,5 \mathrm{~mm}$ y tornillos monocorticales de $5 \mathrm{~mm}$, en la zona resecada se fijó un bloque de beta fosfato tricálcico y estabilizado con tornbillo de 1,2 $\mathrm{mm}$. Se sacrificaron los animales a los 2 meses. Todos los animales sobrevivieron al procedimiento y se pudo llevar a cabo la resección y reconstrucción en todos ellos. No se presentaron signos ni síntomas de infección en el sitio quirúrgico mientras duró el experimento. El tiempo promedio de cirugía fue de 68 minutos. El peso promedio de los animales fue de $3925 \mathrm{~g}$, la pérdida promedio de peso fue de $2,03 \%$. No se realizó eutanasia de ningún animal por motivos de sufrimiento. Al examen macroscópico de las muestras extraídas se observó la presencia de todos los elementos de osteosíntesis en posición, así como continuidad de la estructura ósea casi en su totalidad en la superficie . La utilización de conejos en modelos de reconstrucción maxilofacial es un modelo probado debido a la similitud de los procesos reparativos, su facilidad de manipulación y cuidados. El modelo propuesto representa una alternativa más cercana a los procesos reconstructivos en cirugía resectiva maxilofacial, debido a que somete los injertos a las fuerzas propias de la masticación y de la función orofacial.
\end{abstract}

PALABRAS CLAVE: Injerto óseo; Scaffold; Ingeniería de tejidos; $\beta$-TCP.

\section{INTRODUCCIÓN}

Injertos óseos en bloque son constantemente utilizados en reconstrucción mandibular. Históricamente, los más utilizados han sido el hueso autógeno, representando una técnica validada, protocolizada y con pronóstico adecuado; sin embargo, la morbilidad de la cirugía del sitio donante exige la búsqueda de otras alternativas reconstructivas (Urken et al., 1991; Mehta \& Deschler, 2004; Fariña et al., 2016).

En el mercado actual existen sustitutos óseos que han superado algunas barreras iniciales para su aplicación (Martinez et al., 2015). Sin embargo, no existe suficiente información que permita establecer algún tipo de material no autógeno como viable de obtener regeneración ósea en condiciones normales del sistema estomatognático humano o en modelos in vivo (Rezwan et al., 2006; Hutmacher et al., 2007).

La utilización de modelos experimentales es aceptada para el estudio de la bioingeniería ósea; la utilización de conejos se debe principalmente por ser éstos biológicamente similar al humano y ser de fácil mantención y almacenaje debido a su tamaño reducido (Lu \& Rabie, 2002; Alfotawei et al., 2014). Sin embargo, los estudios experimentales generalmente se realizan en modelos de defectos pequeños en calota (Cantín et al., 2015; Rodriguez et al., 2016) o modelos de defectos mayores en animales de mayor tamaño como perros, cabras o primates (Yamauchi et al., 2008; Masaoka

\footnotetext{
${ }^{1}$ Programa Doctorado en Ciencias Médicas, Facultad de Medicina, Universidad de La Frontera, Temuco, Chile.

${ }^{2}$ División de Cirugía Oral y Maxilofacial, Facultad de Odontología, Universidad de La Frontera, Temuco, Chile.

${ }^{3}$ Unidad de Cirugía Maxilofacial, Hospital Hernán Henríquez Aravena, Temuco, Chile.

${ }^{4}$ Centro de Excelencia en Estudios Morfológicos y Quirúrgicos, Universidad de La Frontera, Temuco, Chile.

${ }^{5}$ Universidad de Tarapacá, Arica, Chile.
} 
et al., 2016). Estos modelos generalmente no comprometen la continuidad ósea, o no se acercan a lo realizado en humanos cuando se realiza una resección y reconstrucción estabilizada mediante elementos de osteosíntesis y sometida al stress de la dinámica mandibular.

El objetivo de este estudio fue desarrollar un modelo experimental de resección y reconstrucción de un defecto mandibular con un bloque aloplástico, estabilizado mediante elementos de osteosíntesis en conejos.

\section{MATERIAL Y MÉTODO}

Se realizó un estudio experimental en modelo animal, en 7 conejos neozelandeses (Oryctolagus cuniculus), machos de 90 días de vida. Se utilizó una hemimandíbula por animal. Los procedimientos quirúrgicos y la mantención de los animales se realizaron en la Sala de Cirugía Experimental y Bioterio del Centro de Excelencia en Estudios Morfológicos y Quirúrgicos, Facultad de Medicina, Universidad de La Frontera, Temuco, Chile.

El protocolo de investigación animal fue aprobado por el Comité Etico-Científico de la Universidad de la Frontera $\left(\mathrm{N}^{\mathrm{o}} 062 / 16\right)$ y se enmarca en los principios bioéticos de las 3 Rs de Russel \& Bursh (Hart, 1995).

Los animales fueron mantenidos en una sala del Bioterio en condiciones de humedad, temperatura y luz estándar; en jaulas individuales, alimentadas con pellet comercial reblandecido y agua ad libitum durante todo el experimento. Se evaluaron diariamente sus condiciones de salud y aceptación de dieta. Todos los procedimientos de manejo de los animales se realizaron bajo las recomendaciones de Guide for Care and Use of Laboratory Animals (National Research Council et al., 2011).

La cirugía se realizó bajo anestesia general intramuscular de Xilazina al $2 \%: 5 \mathrm{mg} / \mathrm{kg}$, Ketamina al $10 \%$ : $40 \mathrm{mg} / \mathrm{kg}$ y Acepromazina al $1 \%: 1 \mathrm{mg} / \mathrm{kg}$. También se realizó bloqueo con anestesia local en zona operatoria con lidocaína $2 \%$ con epinefrina 1:100.000 en dosis de $4 \mathrm{mg} / \mathrm{kg}$.

Luego de rasurar la región submandibular izquierda, se realizó antisepsia de zona operatoria con povidona, y bajo magnificación se realizó una incisión de $3 \mathrm{~cm}$ de largo (Fig. 1); se disecó por planos hasta el periostio, para posteriormente efectuar el decolamiento subperióstico de la región del cuerpo mandibular (Fig. 2). Se realizó osteotomía con sistema piezoeléctrico Piezotome (Satelec ${ }^{\circledR}$,

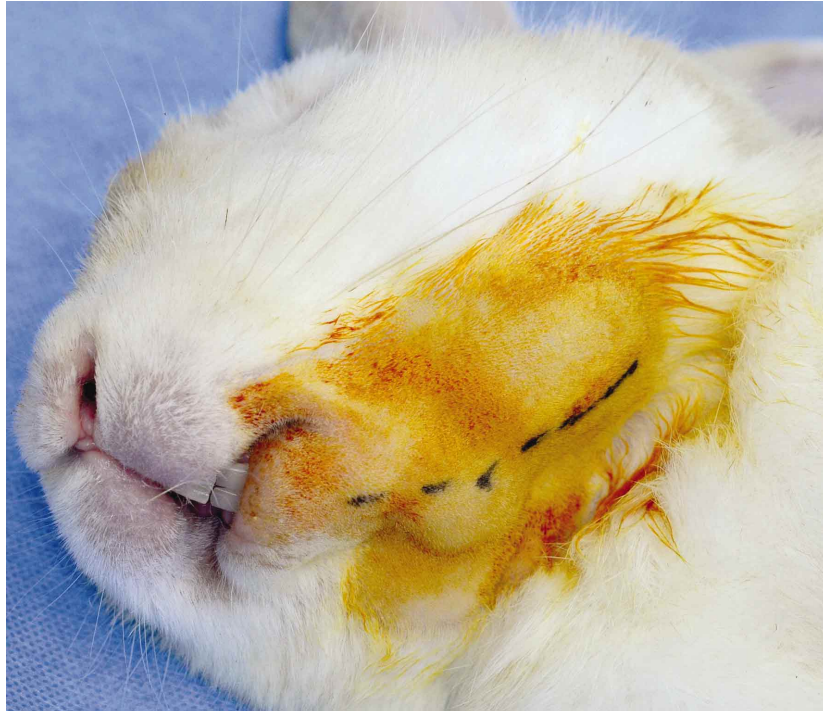

Fig. 1. Preparación y marcaje de zona de abordaje quirúrgico.

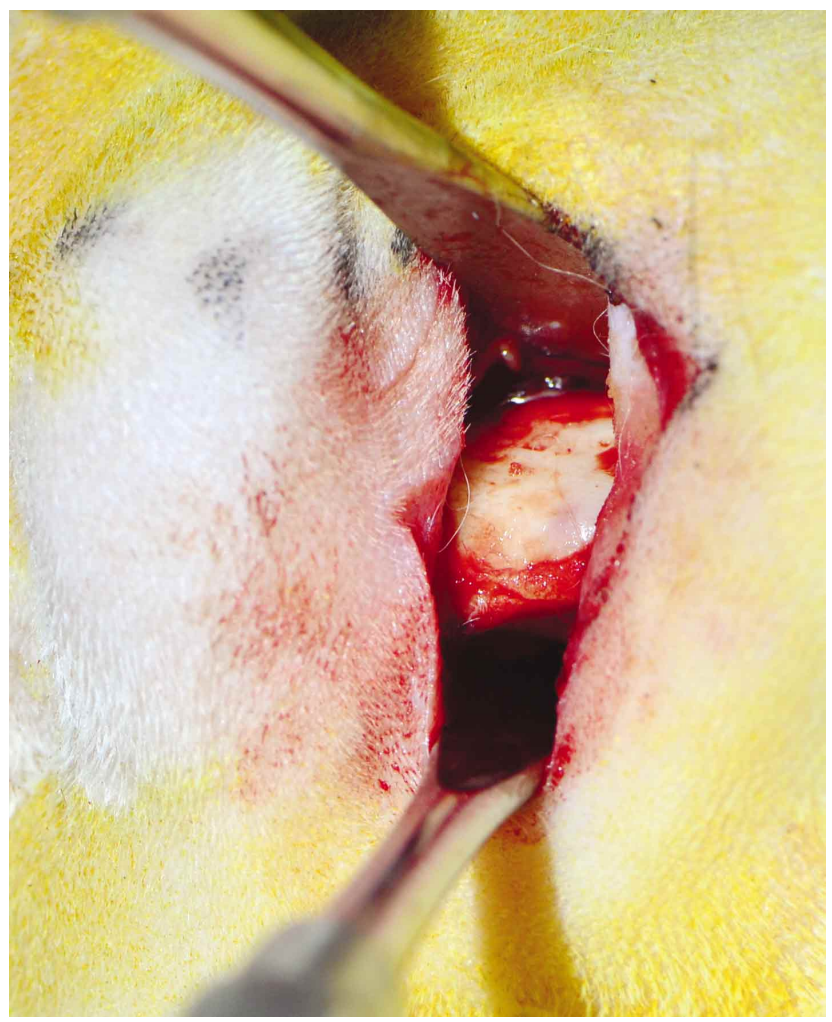

Fig. 2. Decolamiento subperióstico, se observa cuerpo mandibular.

Francia) (Fig. 3) de $5 \mathrm{~mm}$ de largo removiendo un segmento completo del cuerpo mandibular. Una vez asegurada la movilidad de los segmentos, éstos se estabilizaron con placa de osteosíntesis y tornillos monocorticales 1,5 (Biomaterials®, Korea). 
Una vez terminada esta etapa se mantuvo la región fisiológico al 0,9\%. El bloque de aloinjerto Chronus Vivify (Synthes ${ }^{\circledR}$, Suiza) de 5 x 5x 10 mm, se instaló inmediatamente extraído el segmento osteotomizado, y se estabilizó con tornillo de 1,2 mm (Fig. 4). Se realizó aseo de la zona con suero fisiológico y sutura por planos del abordaje, profundos con Vicryl 4-0 y piel con Ethilon 4-0.

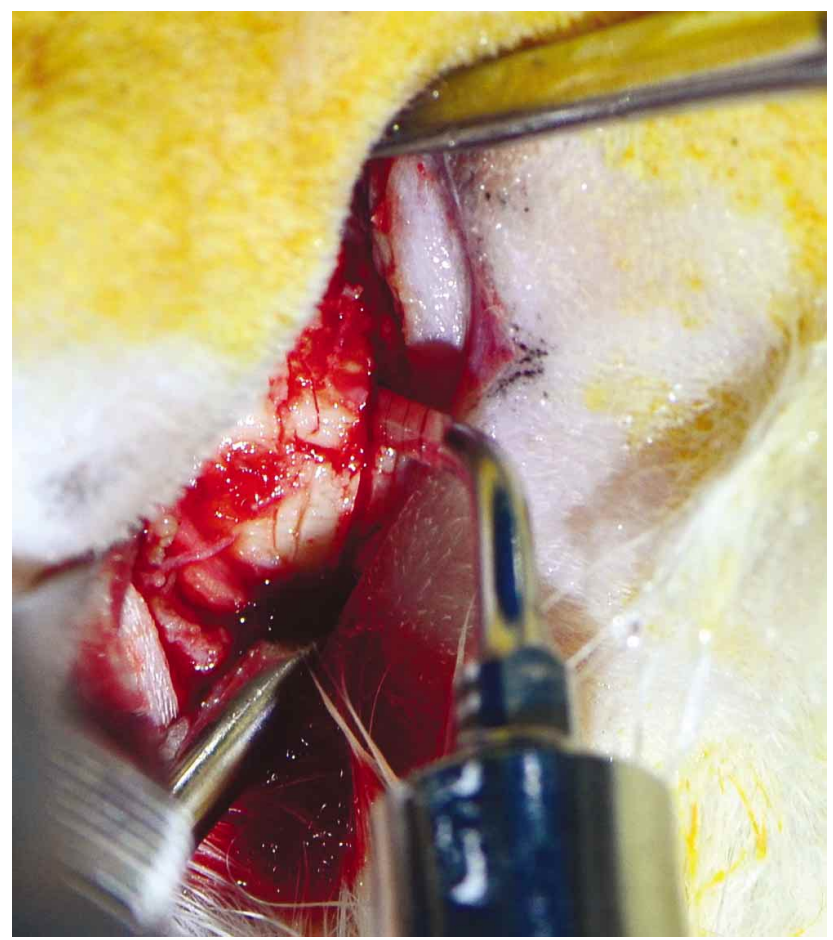

Fig. 3. Osteotomía con sistema piezoeléctrico Piezotome (Satelec, Francia).

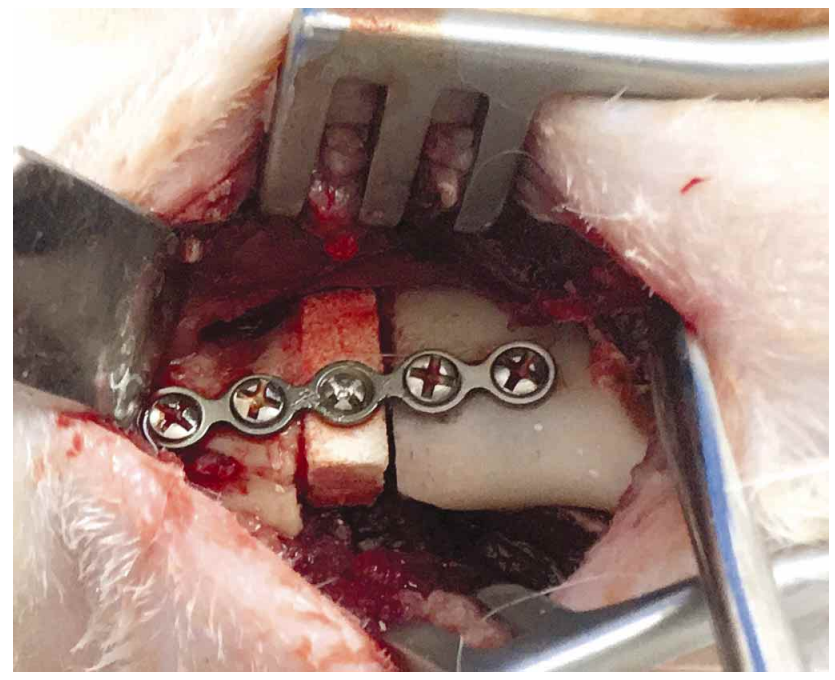

Fig. 4. Bloque de b-TCP estabilizado con osteosíntesis en zona resecada.
Se utilizó en la etapa intraoperatoria y postoperatorio analgesia Meglumine $1,1 \mathrm{mg} / \mathrm{kg}$ y como antibioterapia Enrofloxacino $5 \mathrm{mg} / \mathrm{kg}$, ambos por vía intramuscular. Se mantuvo este esquema farmacológico por 5 días. Se utilizó el protocolo de supervisión de animales de experimentación de Morton \& Griffiths (1985) en la cual se cuantificó el dolor y aflicción causados por un determinado procedimiento para poder tomar las medidas apropiadas de alivio.

Los criterios de interrupción del estudio antes de su finalización, serían la aparición de cualquier patología infecciosa (bacteriana, viral o micótica) que afectase a los animales en experimentación. Si esto ocurría, la totalidad de los animales serían eutanasiados. Además, si en la evaluación diaria se registrase que en alguno de los parámetros seleccionados para la evaluación del estado de los animales estuviese muy afectado (posturas sugerentes de dolor, pilo-erección, agresividad, cambios en el comportamiento social a la manipulación) o superando una puntuación de 9, estos animales serían inmediatamente separados del grupo de estudio y se procedería a su eutanasia . Se realizó la eutanasia de los animales a las 8 semanas post cirugía reconstructiva utilizando sobredosis de ketamina y xilacina.

\section{RESULTADOS}

Todos los animales sobrevivieron al procedimiento y se pudo llevar a cabo la resección y reconstrucción en todos ellos. Mientras duró el experimento no se presentaron signos ni síntomas de infección en el sitio quirúrgico. El tiempo promedio de cirugía fue de 68 minutos (Tabla I), tomado desde la primera incisión hasta el cierre final de la herida en piel. El peso promedio de los animales fue de $3.925 \mathrm{~g}$, la pérdida promedio de peso fue de 2,03\% promedio (Tabla II). No se realizó eutanasia por motivos de sufrimiento. Al examen macroscópico en las muestras

Tabla I. Promedio de tiempo quirúrgico (medido desde comienzo incisión y hasta último punto de sutura)

\begin{tabular}{cc}
\hline Animal & Tiempo total (min) \\
\hline 1 & 90 \\
2 & 70 \\
3 & 60 \\
4 & 65 \\
5 & 60 \\
6 & 75 \\
7 & 60 \\
Promedio & $\mathbf{6 8}$ \\
\hline
\end{tabular}


extraídas se observó la presencia de los elementos de osteosíntesis en posición, así como continuidad de la estructura ósea casi en su totalidad en la superficie vestibular (Fig. 5), en la superficie lingual se apreció continuidad del tejido óseo con un línea central donde se encontraba el bloque de $\beta$-TCP (Fig. 6).

Tabla II. Promedio de pesos al comienzo de experimento y después de eutanasia.

\begin{tabular}{ccc}
\hline Peso inicial $(\mathbf{g})$ & Peso final $(\mathbf{g})$ & $\begin{array}{c}\text { \% pérdida de } \\
\text { peso }\end{array}$ \\
\hline 3720 & 3662 & 1,56 \\
3950 & 3900 & 1,27 \\
3780 & 3700 & 2,12 \\
4000 & 3950 & 1,25 \\
3980 & 3850 & 3,27 \\
4050 & 3960 & 2,22 \\
4000 & 3900 & 2,50 \\
& Promedio & $\mathbf{2 , 0 3}$ \\
\hline
\end{tabular}

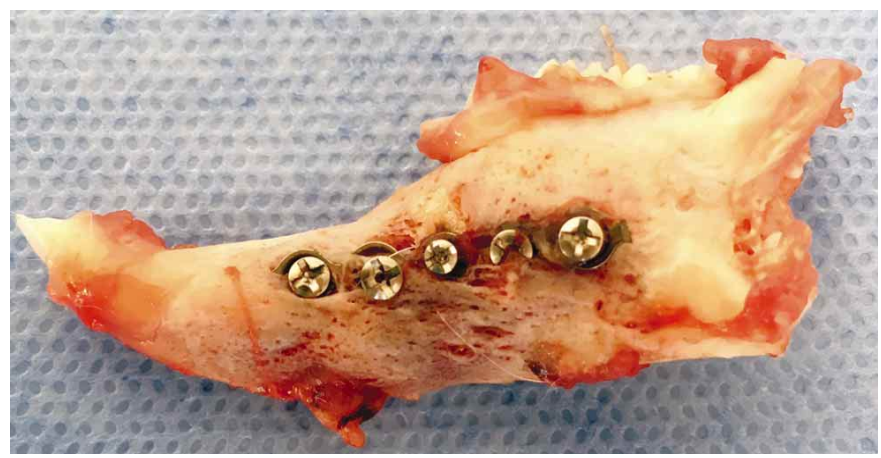

Fig. 5: Vista macroscópica de superficie externa (vestibular) donde se observa continuidad ósea en zona del injerto instalado.

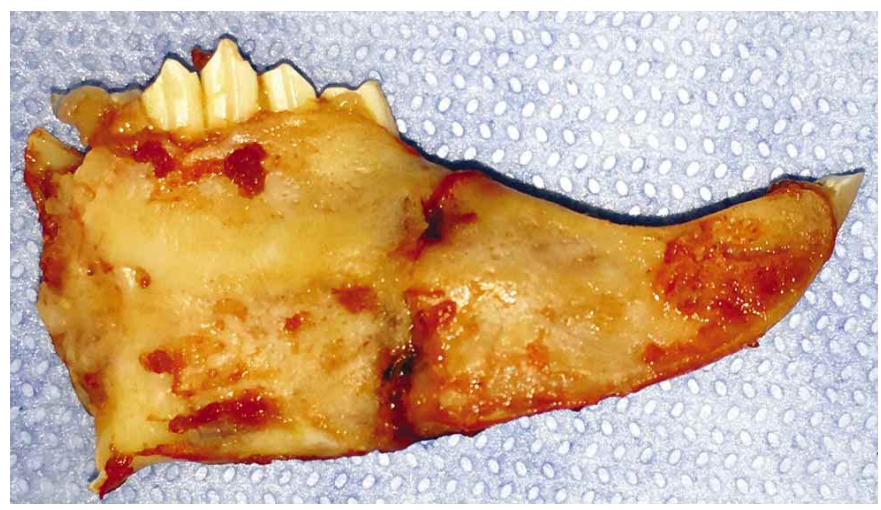

Fig. 6: Vista macroscópica de superficie interna (lingual) donde se observa continuidad ósea en zona del injerto instalado.

\section{DISCUSIÓN}

La reconstrucción de defectos en el territorio maxilofacial sigue siendo un desafío para los cirujanos, quienes han intentando realizar reconstrucciones mandibulares por más de un siglo. A pesar del gran desarrollo, particularmente en los últimos 40 años, la solución ideal implica la reconstrucción anatómica con la suficiente cantidad de tejido óseo y la inserción adecuada de la musculatura perimandibular para lograr una función normal, que aún no se ha podido obtener (Goh et al., 2008).

La reconstrucción de defectos en base a bloques de sustitutos óseos, representa una alternativa que requiere de una evaluación y estudio acucioso antes de indicarlos en grandes reconstrucciones. Así, los estudios experimentales en animales, son una alternativa para la evaluación de estos procedimientos.

El modelo de reconstrucción realizado en base a una resección en bloque y estabilizada con elementos de osteosíntesis, tal como se hace en cirugía de tumores mandibulares en humanos, somete al injerto a las fuerzas masticatorias y a la dinámica mandibular que tiene una directa relación con el comportamiento del injerto en el sitio de inserción. A diferencia de otros modelos experimentales de grandes defectos en mandíbula de conejos, en los cuales los segmentos remanentes son estabilizados de manera trans mandibular (Alfotawei; Naudi et al., 2012), no entrega una realidad cercana a lo que se realiza en cirugía reconstructiva tumoral.

Al evaluar el modelo experimental se observó que los animales no tuvieron complicaciones post operatorias y no hubo que realizar eutanasia, así también el promedio de pérdida de peso fue menor al $3 \%$ de peso total. Cuando analizamos otros modelos experimentales de reconstrucción ósea en animales, encontramos un número reducido de modelos de resección en bloque y éstos se basan, principalmente, en cirugía de huesos largos y en animales de mediano tamaño como perros, caprinos, porcinos o primates (Yamauchi et al., 2010; Masaoka et al.; Tomco et al., 2017). Estos modelos en animales son útiles ya que debido a su tamaño, el procedimiento en general (cirugía, bloques y elementos de osteosíntesis) es más cercano a la realidad de lo que ocurre en humanos, sin embargo por el mismo tamaño de los animales su manejo es más complejo que la utilización de animales de pequeño porte como son los conejos; en este sentido, el análisis bioético es fundamental y parte central al diseñar y justificar un estudio experimental en animales (Hart; Ormandy \& Griffin, 2016). 
Debe estar siempre asegurado que los animales no sufran, que se justifiquen los diseños y la mantención en condiciones óptimas, mejorando el bienestar del animal de experimentación (Russel \& Bursh) (Hart; Fenwick et al., 2011; Chadwick et al., 2016). Así mismo, debe estar siempre resguardado su cuidado de manera acuciosa por personal debidamente entrenado para ello (Krege, 1995), garantizando la supervisión y rápida atención de los animales ante cualquier emergencia o complicación. El entorno de los animales es un factor importante a considerar, ya que su mantención y cuidados sanitarios básicos dependen, de gran manera, como sea el lugar (jaula) donde se alojen, la adecuada ventilación y la higiene y manejo de residuos orgánicos; en este sentido la utilización de conejos permite mantener un número adecuado en espacios reducidos, manteniendo su cuidado estricto sin alteraciones (Stricklin, 1995).

Podemos concluir que la utilización de conejos en modelos de reconstrucción maxilofacial es un modelo probado en muchos estudios, debido a la similitud de los procesos reparativos, su facilidad de manipulación y cuidados. El modelo propuesto representa una alternativa más cercana a los procesos reconstructivos en cirugía resectiva maxilofacial, debido a que somete los injertos a las fuerzas propias de la masticación y de la función orofacial.

\section{AGRADECIMIENTOS}

Los autores agradecen a la Dirección de Investigación de la Universidad de La Frontera por su financiamiento a través del proyecto DIUFRO DI17-0026.

Los autores Juan Pablo Alister Herdener y Francisca Uribe Fenner desean agradecer a La Comisión Nacional de Investigación Científica y Tecnológica, CONICYT, por financiar sus estudios de Doctorado. CONICYT-PCHA/ doctoradonacional/2015-21150598 \& CONICYT-PCHA/ doctoradonacional/2015-21150752.

ALISTER, J. P.; VEUTHEY, C.; URIBE, F. ; VÁSQUEZ, B.; DEL SOL, M. \& SERGIO OLATE, S. Experimental model for the study of mandibular reconstruction. Options in rabbits Oryctolagus cuniculus. Int. J. Morphol., 35(3):1185-1190, 2017.

SUMMARY: The purpose of this study was to evaluate the surgical technique proposed for studies of resection and mandibular reconstruction in experimental models in rabbits. Seven rabbits (Orictolagus cuniculus) were used, in which the left mandibular body was resected, once the mobility of the segments was assured, reconstructed with $1.5 \mathrm{~mm}$ titanium osteosynthesis plate and monocortical screws of $5 \mathrm{~mm}$, in the resected area a block of beta-tricalcium phosphate was stabilized and stabilized with tornbillo of $1.2 \mathrm{~mm}$. The animals were sacrificed at 2 months. All animals survived the procedure, and resection and reconstruction were performed in all of them. There were no signs or symptoms of infection at the surgical site during the experiment. The average length of surgery was 68 minutes. The average weight of the animals was $3.925 \mathrm{~g}$, the average weight loss was $2.03 \%$. No animal was euthanized for reasons of suffering. Macroscopic examination of the extracted samples showed the presence of all elements of osteosynthesis in position, as well as continuity of the bone structure almost entirely on the surface. The use of rabbits in models of maxillofacial reconstruction is a proven model due to the similarity of the reparative processes, their ease of manipulation and care. Our proposed model represents a closer alternative to the reconstructive processes in maxillofacial resective surgery, because it subjects the grafts to the forces of chewing and orofacial function.

KEY WORDS: Bone graft; Scaffold; Tissue engineering; $\beta$-TCP.

\section{REFERENCIAS BIBLIOGRÁFICAS}

Alfotawei, R.; Naudi, K. B.; Lappin, D.; Barbenel, J.; Di Silvio, L.; Hunter, K.; McMahon, J. \& Ayoub, A. The use of TriCalcium Phosphate (TCP) and stem cells for the regeneration of osteoperiosteal critical-size mandibular bony defects, an in vitro and preclinical study. $J$. Craniomaxillofac. Surg., 42(6):863-9, 2014.

Cantín, M.; Olate, S.; Fuentes, R. \& Vásquez, B. Alveolar ridge conservation by early bone formation after tooth extraction in rabbits. A histomorphological study. Int. J. Morphol., 33(1):369-74, 2015.

Chadwick, R. Humans, animals and the scope of bioethics. Bioethics, 30(4):220, 2016.

Fariña, R.; Alister, J. P.; Uribe, F.; Olate, S. \& Arriagada, A. Indications of free grafts in mandibular reconstruction, after removing benign tumors: Treatment algorithm. Plast. Reconstr. Surg. Glob. Open., 4(8):e845, 2016.

Fenwick, N.; Danielson, P. \& Griffin, G. Survey of Canadian animal-based researchers' views on the Three Rs: replacement, reduction and refinement. PLoS One, 6 (8):e22478, 2011.

National Research Council; Committee for the Update of the Guide for the Care and Use of Laboratory Animals \& Institute for Laboratory Animal Research. Guide for the Care and Use of Laboratory Animals. 8th ed. Washington D. C., National Academies Press, 2011.

Goh, B. T.; Lee, S.; Tideman, H. \& Stoelinga, P. J. W. Mandibular Reconstruction in Adults: A Review. Int. J. Oral Maxillofac. Surg., 37(7):597-605, 2008.

Hart, C. B. Legal Control of Use of Animals for Scientific Purposes. In: Tuffery, A. A. (Ed.). Laboratory Animals. An Introduction for Experimenters. 2nd ed. Chichester, Wiley, 1995. pp.37-65.

Hutmacher, D. W.; Schantz, J. T.; Lam, C. X.; Tan, K. C. \& Lim, T. C. State of the art and future directions of scaffold-based bone engineering from a biomaterials perspective. J. Tissue Eng. Regen. Med., 1(4):245-60, 2007

Krege, M. D. Training Materials for Animal Facility Personnel: AWIC Quick Bibliography Series. Beltsville, National Agricultural Library, 1995. pp.95-108.

Lu, M. \& Rabie, A. B. The effect of demineralized intramembranous bone matrix and basic fibroblast growth factor on the healing of allogeneic intramembranous bone grafts in the rabbit. Arch. Oral Biol., 47(12):83141,2002 
Martinez, A.; Balboa, O.; Gasamans, I.; Otero-Cepeda, X. L. \& Guitian, F. Deproteinated bovine bone vs. beta-tricalcium phosphate as bone graft substitutes: histomorphometric longitudinal study in the rabbit cranial vault. Clin. Oral Implants Res., 26(6):623-32, 2015.

Masaoka, T.; Yoshii, T.; Yuasa, M.; Yamada, T.; Taniyama, T.; Torigoe, I.; Shinomiya, K.; Okawa, A.; Morita, S. \& Sotome, S. Bone defect regeneration by a combination of a b-Tricalcium Phosphate scaffold and bone marrow stromal cells in a non-human primate Model. Open Biomed. Eng. J., 10:2-11, 2016.

Mehta, R. P. \& Deschler, D. G. Mandibular reconstruction in 2004: an analysis of different techniques. Curr. Opin. Otolaryngol. Head Neck Surg., 12(4):288-93, 2004.

Morton, D. B. \& Griffiths, P. H. Guidelines on the recognition of pain, distress and discomfort in experimental animals and an hypothesis for assessment. Vet. Rec., 116(16):431-6, 1985.

Naudi, K. B.; Ayoub, A.; McMahon, J.; Di Silvio, L.; Lappin, D.; Hunter, K. D. \& Barbenel, J. Mandibular reconstruction in the rabbit using betatricalcium phosphate (b-TCP) scaffolding and recombinant bone morphogenetic protein 7 (rhBMP-7) - Histological, radiographic and mechanical evaluations. J. Craniomaxillofac. Surg., 40(8):e461-9, 2012.

Ormandy, E. H. \& Griffin, G. Attitudes toward the use of animals in chronic versus acute pain research: results of a web-based forum. Altern. Lab. Anim., 44(4):323-35, 2016.

Rezwan, K.; Chen, Q. Z.; Blaker, J. J. \& Boccaccini, A. R. Biodegradable and bioactive porous polymer/inorganic composite scaffolds for bone tissue engineering. Biomaterials, 27(18):3413-31, 2006.

Rodriguez, R.; Olate, S.; Pozzer, L.; Cantín, M. \& Albergaria-Barbosa, J. $\mathrm{R}$. Tissue response in treated defects with bovine or human bone matrix. Int. J. Morphol., 34(1):351-5, 2016.

Stricklin, W. R. Space as enviromental enrichment. Lab. Anim., 24(4):249, 1995.

Tomco, M.; Petrovova, E.; Giretova, M.; Almasiova, V.; Holovska, K.; Cigankova, V.; Jenca, A. Jr.; Jencova, J.; Jenca, A.; Boldizar, M.; Balazs, K. \& Medvecky, L. In vitro and in vivo study of microporous ceramics using MC3T3 cells, CAM assay and a pig animal model. Anat. Sci. Int., 92(4):569-80, 2017.

Urken, M. L. Composite free flaps in oromandibular reconstruction. Review of the literature. Arch. Otolaryngol. Head Neck Surg., 117(7):724-32, 1991.

Yamauchi, K.; Takahashi, T.; Funaki, K.; Hamada, Y. \& Yamashita, Y. Histological and histomorphometrical comparative study of b-tricalcium phosphate block grafts and periosteal expansion osteogenesis for alveolar bone augmentation. Int. J. Oral Maxillofac. Surg., 39(10):1000-6, 2010.

Yamauchi, K.; Takahashi, T.; Funaki, K. \& Yamashita, Y. Periosteal expansion osteogenesis using highly purified beta-tricalcium phosphate blocks: a pilot study in dogs. J. Periodontol., 79(6):999-1005, 2008.

\author{
Dirección de Correspondencia \\ Juan Pablo Alister Herdener \\ Unidad de Cirugía Oral y Maxilofacial \\ Facultad de Odontología, Universidad de La Frontera \\ Doctorado en Ciencias Médicas \\ Facultad de Medicina \\ Universidad de La Frontera \\ Avenida Francisco Salazar 01145 \\ Temuco - CHILE
}

Email: juan.alister@ufrontera.cl

Recibido : 11-04-2017

Aceptado: 25-06-2017 